\title{
Ecosystem model for healthcare platform
}

\author{
N VISWANADHAM \\ Indian Institute of Science, Bangalore 560012, India \\ e-mail: n.viswanadham@gmail.com
}

MS received 10 August 2021; revised 17 August 2021; accepted 17 August 2021

\begin{abstract}
In this paper, I propose the platform business model to transform the classical Hospital model using the new digital technologies. The ecosystem framework is developed for healthcare service sector where all the stake holders including the core health providers (Hospitals, Pharma manufacturers), Complementors (Diagnostic labs, Pharmacies, Startups), Homecare providers, Software providers, Social media, R\&D labs, Medical schools, Yoga and Gym centers, Government agencies, Patients, etc., using digital technologies to emerge as a highly connected collaborative network. The service chain provides healthcare services via primary healthcare centers, community healthcare centers, public healthcare centers, multi-specialty healthcare centers, emergency healthcare centers, Telemedicine, and mobile and rural health camps. The platform model for the entire healthcare ecosystem is developed and the performance measures, risk mitigation strategies, innovation and finally the platform governance mechanisms are highlighted. This research is aimed at helping the community to be future ready.
\end{abstract}

Keywords. Healthcare; hospitals; healthcare service chain; digital technologies; business models; healthcare platform; Governance.

\section{Introduction}

Healthcare, and more precisely hospitals, are undergoing full scale digital transformation. The underlying benefits are many - improved patient reach and health outcomes, reduced overall costs and increased competitiveness. Higher percentage of younger population, rising income levels, ageing population, growing health awareness, and changing attitude towards preventive healthcare, etc. are expected to boost healthcare services demand in future. Also, focus is shifting from health care to health and well-being. In the future, there will be a greater focus on promoting healthy lifestyles, vitality, and wellness; on primary and secondary prevention; and on early diagnosis. It is important for India to take this opportunity to design Healthcare platform models.

New technologies such as mobile Internet, IOMT (Internet of medical technologies), Cloud, Big Data Analytics, 3D printing, Blockchain, Digital Valets, Drones, and Robots are creating new healthcare industry giants. Use of machine learning and $\mathrm{AI}$ is becoming the new norm for predictive and prescriptive decision making both in healthcare and other Industries. In management terms, competition for the health care Industry will come from ICT and data analytics companies. Start-ups in every area such as EdTech, Fintech, Healthcare and entertainment are disrupting well-established institutions such as shopping malls, banks, hospitals, movies, universities, etc. [1-6]. We are currently witnessing the industry 4.0 in auto, real estate, IT, Finance, and several other sectors [7]. The flourishing companies follow the open platform-based business models collaborating with ecosystem partners. Both Google and Microsoft are collaborating with hospitals and funding startups in healthcare. Their current attention is in the use of AI, ML, Blockchain and NLP in decision making using big data. We will see the Healthcare industry transition to 4.0 soon.

Currently, a limited number of medical practitioners are found in nursing homes and small hospitals. The transition to healthcare 4.0. involves Electronic medical records, digitation of patient data, Cloud, Mobile Internet, IOMTs and connected patients, Big data health analytics and AI, ML and block chain. Innovation in diagnosis and medical treatment and developing personalized medicine are the main focus $[4,8,10]$. The technological transformation may take time like other technological transformations in industry 4.0 [11]. It will be wise to be future ready.

'Health is More than Healthcare' is the principle followed in healthcare 4.0. Current Focus in health care is Disease treatment. Future is on Health and Well-being by Promoting Healthy Lifestyles, Vitality, and Wellness, primary and secondary prevention, and Early Diagnosis. Spiritual, mental, and emotional components are also included in "Health". 


\subsection{National digital health mission (NDHM)}

In August 2020, the Ministry of Health and Family Welfare, established the National Digital Health Mission (NDHM) to provide support for integration of digital health infrastructure [2]. The aim of the mission is to create digital infrastructure: Health ID —an ID used to uniquely identify people, authenticate them, and thread their health records; Digi-Doctor-a comprehensive repository of all doctors practicing or teaching modern/traditional systems of medicine, Health facility registry and Patient health records; and Electronic Medical Records - a digital version of a patient's treatment history from a single facility, and several others.

The digital healthcare market in India is segmented into Tele-health, mHealth, electronic health records/electronic medical records (EHR/EMR), remote diagnostics and healthcare analytics, etc. [12]. The mHealth segment is expected to dominate the market by 2024 , as large number of people are likely to use health and fitness apps to track and monitor daily activities. Advancements in mHealth include wearable health bands that track health in real-time, scheduling doctor appointments using a mobile app, availing reports online, consulting a specialist over a video call, and carry medical history and reports in a healthcare portal on a smartphone [13].

With focus on digitisation of medical records, the Blockchain [14] would assist in providing increased and secure control to patients over their medical records. Also, increasing coverage under public schemes such as Ayushman Bharat, blockchain could be leveraged for automatic verification and authorisation, avoiding back-and-forth between multiple parties, and making claim processing faster and efficient. The Personal Data Protection Bill, draft Digital Information Security in Health Care Act (DISHA) and other such regulations will demand stringent compliances and safeguarding of personal and medical records by healthcare providers.

The primary aim in this paper is to Design a cooperative of Digital health care ecosystem-based Platform with incumbents, startups, R\&D laboratories, medical schools and nontraditional health care players. In this paper, the ecosystem framework is developed for Healthcare service sector that includes all the stake holders and new technologies to become highly connected collaborative networks. The ecosystem model which gives a 360-degree overview of the entire healthcare system and the step-bystep governance mechanism to plan, schedule, monitor and control the patient journey through the healthcare platform.

The topics are outlined as follows. In section 2, the business model is defined and the business model patterns are identified. In section 3, the current hospital service chain business model is described. In section 4, the new technologies are identified. Which include IOT, Big data, AI and Blockchain and their application to healthcare. In section 5, the e-health in India, telemedicine, and current investments in this domain and the start-ups currently in operation are dealt with. In section 6 , the healthcare ecosystem is presented. In section 7, the platform business models and their elements, customer value proposition, actor network, resources, delivery mechanisms are provided. Section 8 deals with the Governance, Risk, Innovations and Performance (GRIP) framework for the Healthcare Platform model. Finally, the conclusions are given in section 9 .

\section{Business model}

A business model (BM) is a conceptual tool that contains a set of elements with prescribed relationships and expresses the logic of earning money. It is a description of the value a company offers to one or several segments of customers: the architecture of the firm, its network of partners and resources for creating, marketing, and delivering this value and relationship capital, to generate profitable and sustainable revenue streams. Thus, a business model is a description of how a company does business and makes money $[9,15]$.

Three main components of Healthcare BM are Customer value proposition (CVP), Value creation formula and Partner network. The CVP identifies the job to fulfil an important need for the patients and deliver value both qualitatively and quantitatively through the partner network. Identifying the customer segments and the delivery mechanisms for each segment is important. The value formula defines how the platform creates value for itself while fulfilling the needs of the patients and the value addition to the partners. It estimates the cost and profit for each customer segment and identifies the revenue model. The partner network that cooperates to produce and deliver products and medical services to the targeted customer segments is an important component of the business model. There is lot of literature on this topic, and it has caught the attention of both academia and Industry. In this paper, the healthcare Platform model is presented in detail.

\subsection{Business model patterns}

Patterns describe proven solutions for recurring problems. Architectural theorist Christopher Alexander introduced the concept of patterns for designing towns, buildings, and constructions. Each pattern describes a problem which occurs over and over again in our environment, and then describes the core of the solution to that problem, in such a way that you can use this solution a million times over, without ever doing it the same way twice. Patterns allow reusing solutions by making them accessible and applicable to others and thus reduce complexity and improve effectiveness and efficiency in problem solving processes [15]. 
Examples of Business Model Patterns include Razor and Blade, Freemium, Long Tail, Sharing Economy, Cloud Business Models such as Software as a Service (SaaS), Platform as a Service (PaaS). Infrastructure as a Service (IaaS), Data-as-a-service, Two-Sided Market, Platform, and many more. Here we consider the platform model for healthcare applications [3]. Platform businesses bring together producers, service providers and consumers in high-value exchanges. Their chief assets are information and interactions, which are also the source of the value they create and their competitive advantage. Platforms create value by facilitating interactions between external producers and consumers. Amazon, Uber, Airbnb are all platform businesses.

\section{Current hospital business model}

The hospital business is owner controlled and asset intensive. The buildings are owned, managed, and maintained by the owner and the organizing committee. The doctors, nurses and other maintenance staff are either employees or on contract. The analysis of Hospital Costs shows that Project costs, land and buildings constitute around 40-50\% of total costs, medical equipment's cost $40 \%$ and rest 10 $20 \%$ for human and operational expenses. With Administrative procedures and bureaucracy, the process of setting up Greenfield might take 3- 5 years. Equipment constitutes around $30 \%$ of all fixed assets; $70 \%$ or more of these devices are imported. Some of the high-cost equipment (MRIs, CT machines) becomes obsolete and need replacement further escalating the costs. Rapid technological changes. increasing costs, limited availability of doctors, inaccurate diagnosis and longer treatment periods are a commonplace. Also, there is lack of direct information sharing between the diagnostic centres, doctors, and patients. Hospitals are to be made more resilient, cost efficient and patient supportive by using new technologies and following new collaborative business models.

\subsection{Current health care service chain}

The patient travel through the health care service chain is known to all of us. All the services, staff and product suppliers are shown in figure 1. The healthcare ecosystem in figure 2, consists of products and front end and back-end services. It is also known that all the companies are not owned by a single organization, but they cooperate to provide end to end service to the patient. The services are healthcare based, transportation or finance based. It is also known that all the companies operate on their software systems, follow regulations and standards. The results, however, are shared on paper. The payment is done for each

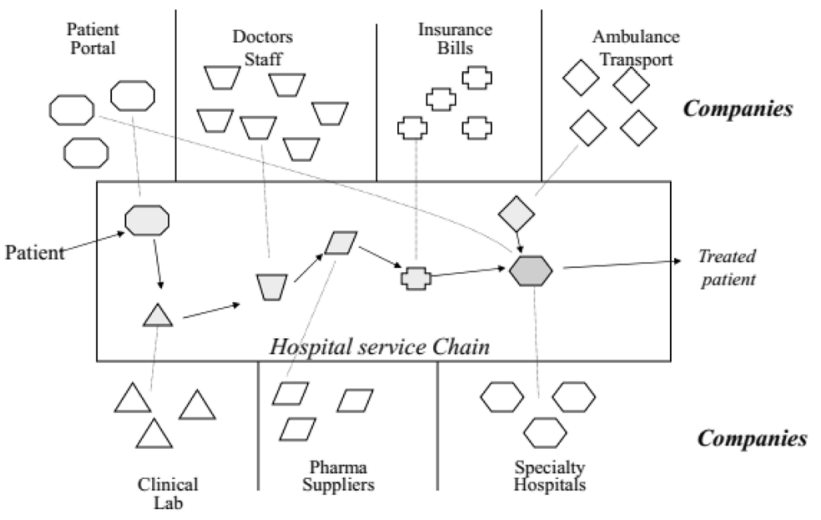

Figure 1. Hospital service Chain.

provider by the patient. In insurance cases, companies could ask the patient to collect the money from insurance company against the bill. The software of each of the services could be interoperable so that the entities can share data and communicate digitally with insurance companies as well with the patient and other partners in the patient electronic health record. Stakeholders lack common outcome measures and shared goals. Many of these actors are well organized and equipped with enough strength to move as they are, rather than going for changed innovations. Uncertainty of success in the new business model is the most common hesitant for most incumbents. Healthcare costs are increasing worldwide while more people are trying to access quality services.

There are specific issues for healthcare industry in India which need attention.

1. In India, till recently, there were more non-infectious diseases than the infectious diseases that spread from one person to another. Non-infectious Diseases are caused by factors such as age, nutritional deficiency, gender, and lifestyle. Examples include hypertension, diabetes, and cancer. They do not spread to others, and they restrain within a person who has contracted them. Alzheimer's, asthma, cataract, and heart diseases are other noninfectious diseases. Infectious Diseases are usually caused by microorganisms called pathogens (fungi, rickettsia, bacteria, viruses, protozoans, worms). When an infected person discharges bodily fluids, pathogens may exit the host and infect a new person (sneezing, coughing, etc.). Examples include Cholera, chickenpox, malaria, Sars, Covid-19, etc. The Covid-19 is highly infectious and has spread worldwide and has created disruptions in several industry sectors and has brought to focus the short falls in the health care sector.

2. There is deficiency of medical practitioners in India. There is one doctor for every 15,000 people and $2 / 3$ of the doctors practice in urban areas. India has $2 / 3$ of its 


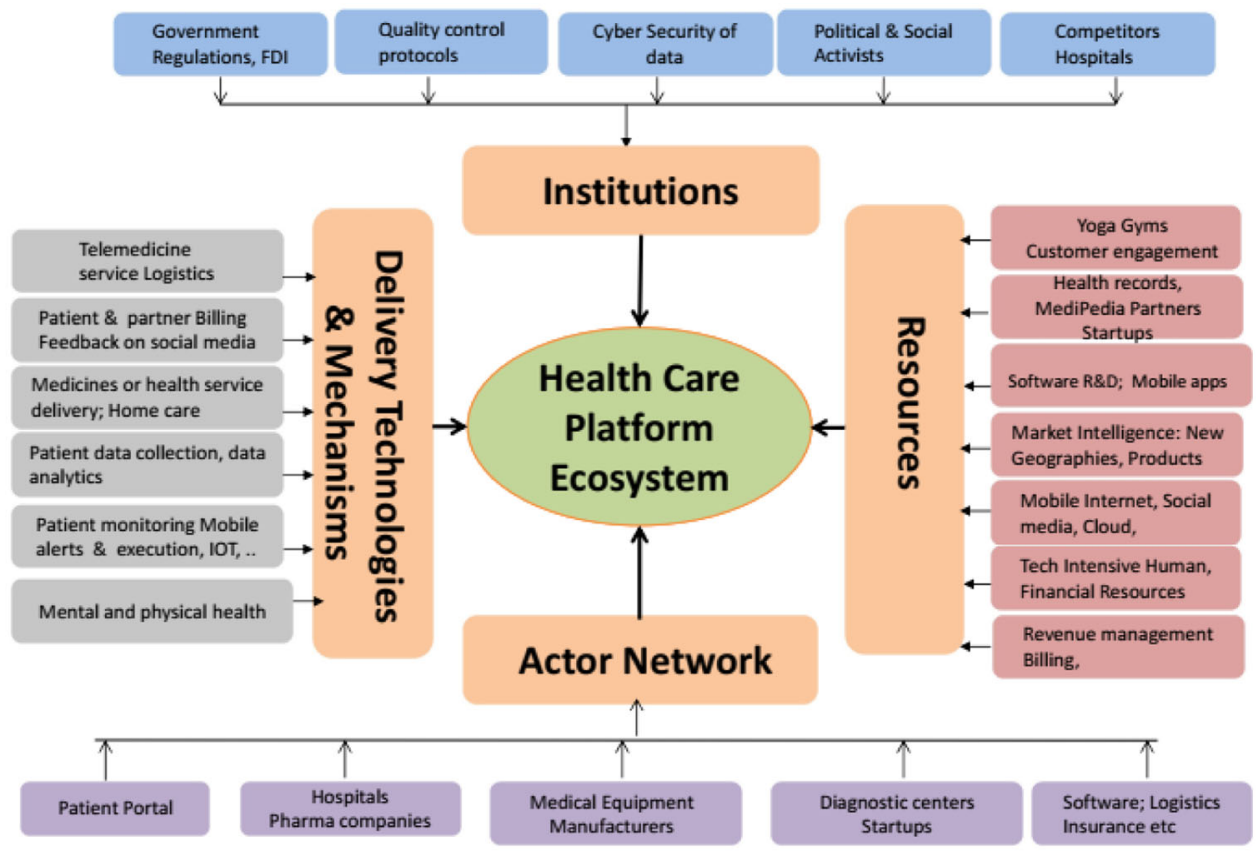

Figure 2. Health care Ecosystem.

population in villages and finding a doctor in villages would be difficult. In times of spread of infectious diseases it becomes difficult to get medical help in villages.

3. Revenue cycle management includes billing, coding, credentialing, denial management, practice management (financial reporting and accounting, human resources and payroll, office management, and purchasing), Group Purchasing for vaccines, insurance, etc. Few large, effective organizations provide billing services and host innovative ancillary services to healthcare practices at a cost below the internal cost. Medical managers need to adopt automation and optimize their processes and systems.

Health is considered more than healthcare. It is time for a social- ecological approach. Up to $60 \%$ of a person's health status is determined by behaviour and social factors, such as socioeconomic status, employment, food security, education, community cohesion and more. While most industries today have years of experience using business intelligence and business analytics tools, the healthcare industry is still in its infancy. Products providing the complete optimal solution for medical practice yet to emerge.

All the above arguments show that the healthcare systems worldwide need a structural change from asset intensive focus on hospitals to developing a collaborative healthcare platform bringing together all the ecosystem players under one roof and provide heath care to the population.

\section{New technologies in healthcare}

Digital Technologies are making their way into the healthcare sector. The Mobile Internet connects doctors and nurses with patients, electronic pill contains sensors that collect information as it travels through gastrointestinal tract, Cloud is used for Healthcare Data storage; Drones deliver medicine, blood, even organs, Robot assisted surgeries are done in private hospitals and are found to be more precise and less painful. 3D printing helps to make Customized medical equipment. It is found that Bones are getting healed 40-80 \% faster when made through 3D printers. Blockchain as a distributed ledger for Secure storage of patient and health information and share with the right partners.

AI helps in precise diagnosis using large sets of clinical data and images for cancer and chronic conditions such as diabetes, cholesterol, cardiac health treatment. NLP and AI help processing Invoices, Bills, and other documents. Big data collected through IOMT and analytics using AI and ML will transform clinical research, treatment protocols increasing virtual care capabilities of health providers.

\section{IOT opportunities for improving healthcare system}

Internet-of-Medical-Things (IoMT) is a network of medical devices that feed vital data in real time to software applications that analyse and communicate with healthcare IT 
systems. Some applications include connecting medical devices, such as ultrasound, thermometers, glucose monitors and electrocardiograms used for tracking patients' overall health; Smart beds in hospitals to circumvent the need of nurses; and Smart medication dispensers at homes that automatically upload information into the cloud; in critical care: IoT-enabled ICUs, emergency care units and operating theatres will enable digital transfer and exchange of patient critical care data to enable better care management by a clinician in real time through remote access.

Also, IOMT allows remote health management for improving the treatment protocols of healthcare ailments by healthcare providers and caregivers using smart devices, and medical devices which provide in real-time of patient health conditions.

\subsection{Big data analytics}

The 4V's represent Volume of data available or collected, Variety of data available- which includes variety in forms, structure and content, the Velocity or speed with which the data is created and processed, and the Veracity of the datawhich deals with the reliability and accuracy of the data that is collected. Vetting the data, cleaning the data, validating the data, and working through the data with healthcare specialists who understand the human side of things is really what must happen. While dealing with patients, one does not have the luxury of making an error. Big data should aid in Healthcare Decision Making. The Big Question is "what data from patients, Medical devices, governments, and local environment should one collect and process to get desired outcomes".

Medical data and decisioning is a complex process. It involves inferring knowledge from complex heterogeneous patient sources, Leveraging the patient/data correlations in longitudinal records, understanding unstructured clinical notes in the right context, and finally Efficiently handling large volumes of medical imaging data and extracting potentially useful information and biomarkers. Analysing genomic data is a computationally intensive task. Combining it with clinical data adds additional complexity. Capturing the patient's behavioural data through several sensors; their various social interactions and communications is also important.

The data can be used in several ways for decision making [16]. Descriptive analytics enables organizations to learn from their past and understand the relationship between variables and how it can influence future outcomes. Predictive analytics techniques are used to answer the question of what will happen in the future or likely to happen, by examining past data trends using statistical, programming and simulation techniques. Prescriptive analytics deals with the question of what should be happening and how to influence it. Prescriptive analytics guides alternative decision using descriptive and predictive analytics, simulation, mathematical optimization, or multicriteria decision-making techniques.

\subsection{AI in health care}

AI will be a major supporting pillar to healthcare sector to complement the effort of doctors - not replacing them. AI is helping for diagnosis and treatment planning for cancer and monitoring of other chronic conditions such as diabetes, cholesterol, fertility issues and cardiac health. Clinical decision support system (CDSS) precisely diagnoses patients using large set of clinical data and images. AI uses smart algorithms to help patients track and understand their health data, assist in medication management, and disperse simple medical advice thus reducing the burden on physicians. AI mines patient's clinical data and scientific literature to gather meaningful medical insights and improving current therapies and creating novel therapies. A combination of wearables and other biomedical devices, combined with AI and ML will transform clinical research, treatment protocols increasing virtual care capabilities of health providers.

Health service providers rely on intermediaries such as clinical, medical bulkers, to operate core functions. AI technologies like natural language processing can extract critical information such as billing amounts, account information, dates, addresses, and parties involved from the sea of unstructured invoice forms received by the company. This will help the accounting teams to process millions of invoices annually from thousands of vendors, partners, or providers.

\subsection{Blockchain in health care}

With digitisation of medical records, the data is shared and stored in cloud. The Personal Data Protection Bill, draft Digital Information Security in Health Care Act (DISHA) and other such regulations demand stringent compliances and safeguarding of personal and medical records by healthcare providers. The Blockchain would assist in providing increased and secure control to patients over their medical records. Also, increasing coverage under public schemes such as Ayushman Bharat, blockchain could be leveraged for automatic verification and authorisation, avoiding document travel back-and-forth between multiple parties, and thus making claim processing faster and efficient.

\section{6. "e-Health" in India}

The digital healthcare market in India is segmented into Tele-health, mHealth, electronic health records/electronic medical records (EHR/EMR), remote diagnostics and 
healthcare analytics, etc. The mHealth segment is expected to dominate the market by 2024, as large number of people are likely to use health and fitness apps to track and monitor daily activities. Advancements in mHealth include wearable health bands that track health in real-time, scheduling doctor appointments using a mobile app, availing reports online, consulting a specialist over a video call, and carry medical history and reports in a healthcare portal on a smartphone.

\subsection{Functions in a digital hospital}

Hospital Digital Platform Stores Patient admission, hospitalization, clinical processes, consultation, referrals. e-prescriptions and e-signatures initiated in the hospital. Orders for MR, X-Ray, ECG, blood, hearing test, etc. are recorded on a computer. All generated data (records, results, invoices, etc.) is archived in a safe Cloud and is accessible to the healthcare staff and patients from anywhere and anytime. Treatment orders and information are processed online using e-signatures and by remote access. Pharmacy, stock tracking and invoicing system can record the entries and exits online. The Closed Loop Medication Administration System ensures that the right drug is administered to the right patient, in right doses at the right time. Activation of emergency Infrastructure such as fire system, security, electricity, water, etc. must be done by a centralized control system.

Digital Hospital administrative, financial, and medical processes are IT based, medical devices are interconnected with each other and with other smart information systems and facility control services. This enhances personnel productivity, improves process quality, and ensures patient safety. Healthcare staff and patients can exchange data inside or outside the hospital for telemedicine and mobile medicine practices.

\subsection{Telemedicine}

Telemedicine uses cloud computing, wireless technologies, electronic health records, hospital management information systems, and tele-care services and they bring in cost-efficiency, reduce consultation time, improve accuracy of treatment and diagnosis, cater to many customers remotely, and maintain high standards of service delivery by providing access to quality care on a real time basis.

Telemedicine enables healthcare professionals to evaluate, diagnose and treat patients situated remotely using ICT. Customised apps contain serialised data about patients' illness and treatment history; these apps also allow patients to schedule appointments, make payments and contact physicians.

Telemedicine has the potential to bridge the disparity in the distribution of doctors and health infrastructure, reduce cost of primary care and improve last mile delivery of quality healthcare. The competitiveness in Tele healthcare services will be defined by digital technologies and data enabled care. Healthcare providers need to embark on digital journey by automating manual processes, digitize medical records, implement IOMT/ AI/ ML and leverage data analytics to enhance customer experience and personalised care. Healthcare providers need to invest heavily in digital technologies in the coming days to stay relevant, engaged, sustainable and successful.

Telemedicine Market: Telemedicine is the most promising solution to healthcare access. The telemedicine market has been largely dominated by Tele-radiology. It involves the electronic transmission of patient's radiographic images such as X-ray, CT scan or MRI from one location to radiologist at another location for interpretation. Tele consultancy is the second largest contributor to the Indian telemedicine industry. Tele-consultancy is audio and video communication between the patient and the doctor or between doctors of different disciplines. Tele-ICU has been regarded as the third largest category in the telemedicine market of India.

Investments in Healthcare: Digital health and technologies fuelled capital investments. In 2019, 53\% angel investments were made in healthcare. In the first quarter of 2020 , the healthcare sector witnessed a $50 \%$ increase in terms of volume (21 deals versus 14 deals in 2019) and $14 \%$ in terms of value (US\$ 452 million versus US\$ 398 million in the first quarter of 2019). Some big deals are Curefit (US\$ 111.5 million), Iora Health Inc. (US\$ 126 million) and HealthCare Global Enterprise Ltd. (\$119.7 million). Medix Global, a London-based innovative digital health management solutions provider is setting up a panIndia digital infrastructure for tele-medicine and offline medical solutions including diagnosis and early disease detection services related to chronic diseases such as cardio-vascular, diabetes management, cancer and stroke.

\section{Healthcare business ecosystem}

Healthcare ecosystem is a network of independent companies working together to gain comparative advantages through symbiotic relationships benefiting all parties. It is a Network Organization with core health providers (Hospitals, Pharma manufacturers), Complementors (Diagnostic labs, Pharmacies, Startups), Homecare providers, Software providers, Social media, R\&D labs, Medical schools, Startups, Yoga and Gym centers, Government agencies, Patients, etc., which delivers Healthcare products or services through both competition and cooperation. Ecosystem thrives when the participants streamline the flow of innovations, talent, and capital throughout the system $[5,17-19]$. 
Ecosystems emerge due to modularity and the interactions and the co-existence of different types of complementarities. There are four types of modularity that are influencing the businesses today. They are product modularity, service modularity, organizational modularity, technological modularity. In products, modularization refers to a product architecture where different functions of a product are implemented by different and relatively independent physical components whose interfaces are defined by a set of interface standards in a way that allow substitutability of components. Similarly, services can be executed independently, and their combination creates value. Modularization of product designs paves the way for similar modularization of organization designs. Technological modularity allows interdependent components of a system to be produced by different producers, with limited coordination. Modularity allows for coordination of independent yet interdependent firms through the ecosystem. Ecosystems require providers of complementary innovations, products, or services belonging to different industries and not be bound by contractual arrangements-but have significant interdependence. Ecosystems encourage alignment through rules of engagement, standards, and codified interfaces.

Health care ecosystem providers can learn from other industries and harness the innovation potential of the ecosystem model and the new technologies to improve access and quality and reduce cost. Health care ecosystems could facilitate new solutions and major improvements in various dimensions by enhancing coordination and effectively using data across partners. Application of Platform ecosystem models in health care is a new trend. Walmart has launched cost-efficient outpatient clinics, and tech players such as Google, Amazon, Microsoft, and Apple are offering health care solutions such as cloud services for health data and telemedicine. There are about 5000 startups currently operational in India. Also, patients are increasingly demanding levels of service and choice in health care that they are receiving in other areas such as banking, retail, etc. New technologies such as big data, IOT, Drones, Blockchains, etc. have created new forms of access and interaction. Secure and cost-effective datasharing solutions are increasingly available and enable new ecosystem applications.

\section{Platform business model}

A business model is a conceptual tool that contains a set of elements with prescribed relationships and expresses the company's logic of earning money. It is a description of the value a company offers to one or several segments of customers; the architecture of the firm, its network of partners and resources for creating, marketing, and delivering this value and relationship capital, to generate profitable and sustainable revenue streams. Thus, a business model is a description of how a company does business and makes money [20, 21].

We define the healthcare ecosystem model of a platform and its main components which are the customer value proposition (CVP), value creation formula, partner network, the other four business model pillars include healthcare service chain processes; key resources; care delivery mechanisms and institutional and social constraints and finally the operational governance. The platform makes money by delivering the health care products/ services using the resources following the social and institutional constraints and delivering to various customer segments the products and services using all the opportunities including the primary, secondary and tertiary centers. We then present the Governance framework. In this paper, we extend our ecosystem framework [22,23], to include the healthcare service perspective and various customer segments and develop the business model ecosystem.

The study of the platform business model became a topic of even greater interest due to the context of Covid-19 pandemic. This global health crisis forced companies to accelerate the digital transformation process and people to live exclusively in the digital world. Therefore, the practical importance of this topic is a motivating factor of the present study. The implications of this study are more directed towards academic research.

\subsection{Customer value proposition (CVP)}

The Actor network has several customer segments: Core and Complementary providers of medical products and healthcare services, $3^{\text {rd }}$ party developers who create boundary resources, such as APIs, software development kits and finally the patients. Each customer segment contributes to the growth of the Ecosystem either directly or indirectly. The CVP identifies the jobs that fulfil important needs for the targeted customers segments and deliver value. Each customer segment has its value proposition and a revenue stream. They could be dependent on each other i.e., results of one segment could be inputs to the other. The platform should create value for each of them. The platform does the Match making: attract producers of medical products and healthcare services and the consumers including the patients who need those products and services making sure of the network effects. Platform costs should be lower than market costs and the matching should be more competitive for both sides.

\subsection{Actor's network}

The actor network is a network of organizations including Patient portal, Hospitals, Laboratories for conducting Dental, CT scans, X-ray, Blood, and other tests, 
pharmaceuticals, medical devices and equipment, health insurance, Diagnostics, Nursing homes, Telemedicine and medical tourism, start-ups, social media, third party service providers such as IT, Cloud, APIs, software companies, distributors, patients, competitors, government agencies, etc., involved in production and delivery of medical products or healthcare services through both competition and cooperation. The third-party developer, on behalf of the platform owner, develops applications, services, or systems for satisfying end-users of the platform.

Platform owner attracts the patients and healthcare partners, connects the customers to the service provider, coordinates and executes the interaction and delivery. Platform acts as an intermediary between the patients and service providers. For each patient requirement, the Platform maps and manages the chain of activities identifying the firms involved.

\subsection{Institutional and societal constraints}

Products and services delivered need to follow regulations on privacy, safety, data protection, labor welfare, insurance, and tax regulations. FDI is permitted up to $100 \%$ under the automatic route in the hospital sector and in the manufacture of medical devices. Recent policy measures like Ayushman Bharat; Decision to investment of $\$ 200 \mathrm{~B}$ in medical infrastructure by 2024 , will also help to drive the growth of India's healthcare sector. The National Digital Health Mission (NDHM) is to provide support for integration of digital health infrastructure. The platform management is responsible for the safety and integrity of the customers/patients and partners, data privacy, billing, etc.

\subsection{Resources}

Resources include Human (Skilled and Research staff), Financial (Banks, VCs, etc.) and Industrial (Pharma Clusters, Logistics), Relationship capital (partner network) and Digital (Cloud, Blockchain, Analytics, Mobile apps...) resources required to deliver the value proposition to the targeted customers. The technology-driven resources such as Internet, Cloud, Mobile, AI, ML algorithms, Big data analytics, Blockchain make high impact. Ordinary resources needed to include salespeople in pharmacies; websites and delivery in e-pharmacy; mobile internet for social media, etc. Practo's user-friendly website, a simple business model and 24/7 customer care service allows people to get healthcare appointments from their homes.

Care Delivery Mechanisms: The function of delivery is to ensure the availability of right healthcare at the right place, in right quality, at the right time and at right cost for the right patients. To achieve this objective, integration and synchronization of material, information and financial flows across all organizations is needed. In case of the platforms, there are several customer segments who are themselves part of the Platform and their timely services are important. Most often, the consumer order follows sequential steps, and they need to be monitored for timely execution. Delivery of healthcare can be made efficient through use of IOT, sensor networks and augmented reality. Coordinating and monitoring the delivery process is an important issue and is often done using an execution dashboard.

Value creation formula: The value creation formula defines how the platform creates value for itself and its partners while fulfilling the needs of the patients and partners. A platform has multiple partners-such as Pharma manufacturers, healthcare service providers, and digital services providers, as well as predictive analytics software and API developers. They are not paid for the development work. Instead, often the developer is offered a marketplace for its applications. The platform owner taps into multiple networks of partners, characterized by heterogeneous innovation capability and knowledge resources following a revenue sharing model.

Building patient volume requires upfront investment in awareness generation and marketing. The providers need to generate volumes of paying customers to achieve profitability across their network. Both technology as well as service providers need access to patient capital for investment in technology, manpower, capacity building and marketing for expansion (figure 3).

\section{Governance: partner selection, coordination and control}

The Orchestrating Platform mobilizes the needed assets of other companies to support its own growth initiative. The platform should get involved in orchestration and run a high-end hospital as a joint venture. The platform owner should keep innovating and make the outsourced model outdated and look for additional or new partners. A combination of wearables and other biomedical devices, combined with AI and ML will transform clinical research, treatment protocols increasing virtual care capabilities of health providers.

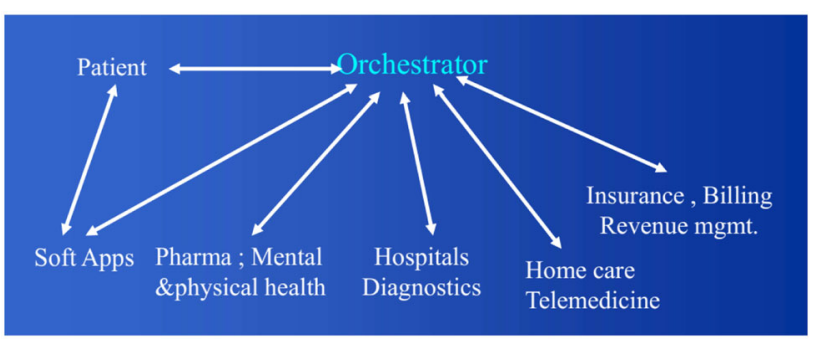

Figure 3. Healthcare Platform Orchestration. 
Platform Assets and Services: The Products of the Platform offers to the clients are Network, Relationships and Knowledge. Its competitive advantage lies in the scope and timeliness of its domain knowledge and in the number and depth of its relationships with its members including the Governments. Platform Providers not only need to enrol doctors to use technology, but recruit and train frontline (nursing, paramedic, maintenance) staff. Availability of such human resource with digital touch is a challenge.

The platform assets that are hard to copy are the community (partner network and patients); Resources its members own and contribute, be the Hospitals or Data analytics or ideas. Because members of the partner network are mutually dependent, good performance by one boost success of others. Platform should provide a definitive asset for its partners to build their presence, to grow their practice or business, and to engage the patients in a way that will provide healthy long life.

A Well-defined governance structure follows the regulations of the government, social groups, labor, and environmental standards, identifies and manages relations with Patients and Healthcare providers, etc., Builds effective communication, collaboration, and coordination among the network partners for growth enhancement, Identifies and categorizes risks from various ecosystem sources and puts in place risk mitigation strategies in operational readiness, For every patient, selects the partners, allocates the tasks and responsibilities to form the network and finally manages a control room for monitoring and execution of the planned activities in a timely manner under both normal and severe conditions.

A separate healthcare service chain is formed for each patient. The chain partner selection based on both Structural features (asset specificity, capabilities) and the relational ties (Staff; Govt., Telehealth, diagnostics, home care, etc.). The second step is Coordination where the tasks assignment and scheduling are done (who does what and when) and communicated to everyone. Finally, in the execution step the order status is monitored so that processes work as per plan and control exceptional events.

Control Tower: Digital command centres/ control towers: Future hospitals could use digital command centres to predict and determine their services (operations, logistics), equipment such as ICUs, oxygen $\left(\mathrm{O}_{2}\right)$ cylinders and clinical/procedural needs, in addition to improving the patient experience. Using predictive analytics, the command centre could analyse hospital (clinical and operational) and community health data to forecast business, experience, and clinical needs. A few applications are as follows.

1. Clinical needs: Digital real-time monitors could track a patient's health in the hospital, and the command centre could use AI and predictive analytics to help with diagnosis and treatment plans.
2. Supply chain, operations, and other logistics: Technologies can predict staffing, supply chain, operations, and logistics needs to ensure efficient use of resources.

3. Patient experience: Another possibility is the patientfacing virtual assistant powered by natural language processing (NLP) and $\mathrm{AI}$ in the command centre/control tower. The device is equipped in each room and takes verbal requests from the patient, uses NLP to process the requests, and then uses $\mathrm{AI}$ to assign them to the most appropriate person (or robot) based upon efficiencies.

4. Pandemic or epidemic disease: Monitoring and tracking of biometrics and test results could identify a potential infectious disease on the rise before it spreads more broadly.

\subsection{Performance of healthcare platform [21]}

The performance of a platform is judged by the number of partners on one side the patients and payers on the other and the satisfaction based on patient health improvement and the profit the partners make. General performance measures include time spent by the patient and the costs involved and the attention they receive. In the longer term, there are other measures that need to be given attention such as traceability, data protection, disease predictability both for the patient and the society, etc.

Performance measurements of the healthcare are critical. The ecosystem wants of its medical suppliers, service providers such as doctors, nurses and other staff, software developers, clinical labs, transport providers and other complementors to follow the regulations on quality, responsiveness, and resilience (the ability to assure continuity of supply despite problems in the supply chain or in the ecosystem). In other words, the patient goes through the service chain and not have to worry about whether the tests, diagnosis and medicines are in the right direction. Often, there could be wrong test results, wrong medicine delivery and wrong diagnosis with incomplete knowledge or testing and the platform should be cautious and appoint a fault diagnostic team to avoid such events.

Traceability of the products and services which runs through several stake holders and finally to the patient. There are billions of pharma products being manufactured everyday globally, through complex supply chains that extend to all parts of the world. However, there is very little knowledge of how, when and where these products were originated, manufactured, and used through their life cycle. Even before reaching the end consumer, goods travel through an often-vast network of retailers, distributors, transporters, storage facilities, and suppliers that participate in design, production, delivery, and sales. It is known that counterfeiting is very prevalent in pharma products. Pharma supply chain visibility and transparency are important for the safety of the patients. Also the clinical 
tests and the results need to be visible to the patient and the doctors.

Managing information and control of transparent interactions about every patient's journey can be a very difficult task. It requires accurate data collection and secure data storage to enable a flow of trusted information between parties. This responsibility is to be borne by the platform owner through centralized information depositories. Relying on one single organization to broker such sensitive and valuable information requires a great deal of trust to be invested by every actor. Such organization will also gain significant power through the possession of this valuable data, which could be misused. Even if this entity can be trusted to be a good actor, it must possess the technical capabilities to store and handle this information effectively and should not become a single point of failure which leaves the whole system vulnerable to failure (e.g. hacking, or corruption). Various incidents in the past decades have shown that even a tight and costly security mechanism cannot guarantee the complete data security, leaving organizations in a network at potential risk.

\subsection{Risks in the platform ecosystem}

In healthcare service, risks like - quality of the medicines; disposal of non-degradable wastes like unused medicines, used needles, syringes and other medical equipment; inefficiencies in handling emergency cases, ineffective handling of cases that can lead to interchanging of records or loss of records, etc. [24, 25]. We need to identify all possible social, political, and environmental risks that may affect the healthcare service chain and the pharma goods and medical equipment supply chains, as well as the information and financial flows. One must identify the risks that the platform as well as the partner companies may face and estimate the risk and determine what it takes for their resolution. The company should have the alternative actions in place. Risk monitoring and mitigation strategies are important. The platform should be resilient and should be able to recover to the working state as soon as possible.

There is a risk of Competition from Existing medical practitioners in areas of operation. They have an established patient base and goodwill in the community. They provide face to face personal communication. Patients still prefer a certain amount of human touch/face to face interaction with the care provider. Platform and Telemedicine players need time and effort to build trust in the community to overcome this challenge. It is important that the existing practitioners are a part of the platform. Uninterrupted power and seamless internet connectivity with sufficient bandwidth, power backup in the form of battery/generator are pre-requisites for telemedicine.

Cybersecurity is a new priority as ransomware attackers hit hospitals and health systems. Hackers stepped up attacks on healthcare companies going beyond phishing attacks, stealing data and information, and selling on dark web. Ransomware attacks shut down IT systems and slowed operations at hospitals and healthcare facilities in some countries. Denial of service (DoS) attacks (deny service to valid users) make Web server temporarily unavailable. How to protect against DoS threats?

There is a need to identify the government risks such as change in the government, state - central government relations, international relations, and corruption. Trade taxes increase, import and export restrictions, etc. can occur as governments change. Social risks need to be identified if the projects involve environmental degradation or land acquisition or people displacement as in infrastructure projects.

Customer Hesitancy for Telemedicine is a big issue that need to be addressed. Patients are used to the physical presence of a familiar doctor and do not feel the same level of comfort, reassurance, or confidence when it comes to a stranger on a screen in a white coat. Employing trained nurses to facilitate consultation can help circumvent such issues. Telemedicine cannot substitute a face-to-face consult in all cases and requires supporting infrastructure to be effective. It needs to be integrated with basic diagnostics e.g., with routine lab tests, with high-quality assessment of basic vitals such blood pressure, with digital health records that can facilitate continuity of care over time, To access medications and Tertiary care facilities in case specialised or advanced treatment is required. Also, Doctors are concerned about medico-legal implications and are reluctant to providing remote advice. These issues need to be addressed.

\subsection{Innovations}

Traditional pharma and medical equipment and health care services innovations are rooted on a standard and wellaccepted model characterized by heavy investments in the discovery processes and invention followed by commercialization in the form of new processes and products. The new-to-the-world inventions are characterized as innovation. Services innovation is about creativity, technology, $\mathrm{R} \& \mathrm{D}$, and entrepreneurship and new ways of doing business and interacting with society. Broadband, mobile and wireless networks, devices and services allow for richer services: communication, commerce, media, health, marketing and security [26]. The global service platforms such as Internet, Facebook, YouTube, Wikipedia, WhatsApp, Telemedicine, Ports, Airports, Suez and Panama canals, etc. provide the key to open the doors for integration and betterment of the world economy. The supply chain or service chains consist of global partners collaborating in serving the customers who are also globally dispersed. Government policies have in many respects been constructive. There has been a co-evolution of public policy 
and firm strategy. One need to formulate an integrative, coevolutionary innovation strategy that would lead to high healthcare industry growth or excellent service quality by working with innovations in several of the ecosystem elements and their convergences to provide easy access to quality healthcare products and services at affordable cost to the people.

There are several innovations in healthcare and other industries that worked successfully in a different industry segment. Open innovation approach follows Innovations created in the same or cross-industry context and have already worked successfully in the market. This assists firms in cutting down uncertainty and risk. In the telemedicine market, the surgeon can travel to the village or town and rent a Procedure Kit for a specific surgery. The kit also includes a full support service, including transportation to and from hospitals, the cleaning of the instruments before and after each surgery and other aspects of "fleet management." One can also learn from The founder of Aravind Eye Care - a company that provides cheap eye care and eye surgery through a network of hospitals in India - found a ready analogue in McDonald's business model: “it [Aravind Eye Care] is run like a McDonald's, with assembly-line efficiency, strict quality norms, brand recognition, standardization, consistency, ruthless cost control, and above all, volume."

In the health care several innovations from retail, manufacturing and services can be adapted for creating efficiencies and customer confidence. In ITC's E-Choupal, Sanchalak (person selected and trained by ITC) acts like a hub or an orchestrator who connects the various stakeholders in the ecosystem. According to social network theory, Sanchalaks fill structural holes [27] (Static holes that can be strategically filled by connecting one or more ties to link together disconnected components). Sanchalaks' key role in innovation diffusion and information dissemination needs to be recognized. Like in E-Chopal, Digital Hospital can create similar intermediaries in the village's nurses, paramedical staff and doctors in districts and district hospitals so that patients feel comfortable to deal with online doctors. Hospital ecosystem connects all such intermediaries. Annual gatherings, health summits with the hospital staff, local doctors and patients will strengthen the platform and creates confidence in the patients and collaborating doctors and staff.

Several people have identified the automotive and aviation industries as lead industries from which they can regularly transfer technologies, processes, and concepts. In the fashion sector Li \& Fung orchestrates the manufacture of fashion products from all over the world. A company's strength lies not as much in the capabilities it possesses but on as much as competencies it can connect to. Capability to connect to competencies - the core competency of network orchestration- is as important as firm specific capabilities.

\section{Conclusions}

Healthcare ecosystems offer several important benefits. They provide fast access to a broad range of external capabilities that may be too expensive or time-consuming to build internally. This is particularly relevant for companies that want to reap the benefits of open innovation, an area where ecosystems outperform current hospital models. Also, Ecosystems can scale much faster than the current vertically integrated healthcare models. Their modular setup, with clearly defined interfaces, makes it easy to add partners and expand the network [11]. Ecosystems offer a large degree of flexibility and resilience. They can quickly adapt to changing patient needs or new technological innovations, which makes them particularly advantageous during times of high uncertainty.

A Digital health care ecosystem-based Platform with traditional and nontraditional health care players will concentrate on promoting healthy lifestyles, vitality, spiritual, mental, and emotional issues and wellness, primary and secondary prevention, and early diagnosis. It is possible to design such a platform and in times to come, it will become a reality. In the future, the connected world must deal with both infectious and noninfectious diseases affecting the physical and mental health. New technologies must be taken to advantage to detect their presence, and transmission potential invent the diagnostics tools and discover pharmaceutical products and their distribution to the needy.

To realize the transformative potential of big data, health care providers and other stewards of patient information must implement effective data governance. The challenges are significant, however, especially for special categories of data such as protected health information and personally identifiable data. Providers must create an efficient process that concurrently addresses a number of complex privacy, cybersecurity, business, and legal considerations. As artificial intelligence (AI) and related capabilities mature, providers face growing opportunities to create clinical, operational, and financial value by sharing patient data with innovative partners. For example, companies such as Flatiron, Tempus, and 23andme have created significant value by applying AI and analytics to patient data. To capture these opportunities, organizations are sharing data across many vendors that provide services to improve patient care.

There is an urgent need to streamline and technologically transform Health Care services to Algorithm assisted Digital health based on real time patient data. Education Institutions and Medical colleges can assist the Government to initiate education, research, and entrepreneurial programs in digital health care sector; Produce Healthcare Practitioners not medical doctors; Re-innovate Pharma manufacturing and Smart Food sectors; Plan and Build Integrated Service Systems: Smart Medi Cities, Smart Healthcare Villages; Start-up Ecosystems, etc. In India for example, Collaboration between technical 
Institutions (IITs and IISc) and the medical schools such as AIMS, Apollo, Manipal, etc. and IT companies such as TCS, Infosys could generate the needed talent and technologies through innovation and development. Student projects, Industry internships and cross industry (medical, IT, Engineering and Bio) are a must for development.

Health care data collection and diagnostics systems software development using this data, electronic medical components and instruments development, training of the doctors and the staff towards digital care need to be undertaken. Thus, Government support and public private partnership is needed for making the initial development a reality and a health care promotion revolution. Multidisciplinary Conferences and Workshops would enable the students, staff and faculty in healthcare, big data, Artificial intelligence and IT to interact and contribute to the development.

But these data-sharing arrangements have attracted increasing scrutiny from the public and regulators. Because regulators have imposed very strict standards, providers generally cannot share protected health information without a patient's consent. Recently available cloud technologies provide streamlined capabilities for establishing and enforcing data-sharing governance. These technologies provide long-term integrated solutions but require enterprise-wide cybersecurity and data protection policies that cover cloud data management models, as well as the ability to manage cloud infrastructure. As technology transforms health care, providers must ensure that patient data is used safely and ethically.

The competitiveness in healthcare services in the next decade will be largely defined by digital technologies and data enabled care. Healthcare platform adoption may follow an $\mathrm{S}$ curve with a slow start, followed by a steep acceleration as technologies mature. The goal of "going digital" is not enough; people must have skills, capacity, and tools to utilize them. Secure Digital Health care should be accessible, affordable, and available to all. The noble prize winner Solow's (1987) Paradox is appropriate here as well. He said, "We see transformative new technologies everywhere but in the productivity statistics". Steam engine, Electricity and computers increased productivity both directly and by complementary innovations, but each has taken decades to develop complementary technologies and products and finally become productive in the economic and customer sense. Similar will be the case with digital platform healthcare. Benefits require complementary co-inventions and investments, in additional technologies (robotics, self-driving cars, intelligent personal assistants, smart health care, etc.) applications, processes, business models, and regulatory policies. In view of the increased connectedness of people and the societies, it may take lesser time. We can hope for healthcare platforms by 2025 .

\section{Acknowledgements}

I thank the Indian National Science Academy (INSA), New Delhi and Indian Institute of Science (IISc), Bangalore for the Honorary Scientist Position.

\section{References}

[1] Randolph G, Marc P and Maulesh S 2017 The hospital of the future: How digital technologies can change hospitals globally, Deloitte Centre for Health Solutions

[2] Ministry of Health \& Family Welfare, Digital Health Initiatives, Government of India, 2017

[3] National Digital Health Mission, 2020, Strategy Overview Making India a Digital Health Nation Enabling Digital Healthcare for all, July 2020, National Health Authority

[4] Sarwal R, Prasad U, Madangopal K, Kalal S; Kaur D, Kumar A, Regy P and Sharma J 2021 Investment Opportunities in India's Healthcare Sector, NITI Aayog. March 2021

[5] Serbanati L D, Ricci F L, Mercurio G and Vasilateanu A 2011 Steps towards a digital health ecosystem. J. Biomed. Inform. 44: 621-636

[6] Viswanadham N 2015 Structural holes and supply chain orchestration. Heavy Haulers 1(2): 82-93

[7] Carmen-Alexandra S and Dragos T 2021 Platform business models - a case study of the technology industry. J. Econ. Manag. Sci. 4(1)

[8] Agarwal R, Dugas M, Gao G G and Kannan P K 2020 Emerging technologies and analytics for a new era of valuecantered marketing in healthcare. J. Acad. Market. Sci. 48(1): 9-23

[9] Business Model Canvas - Wikipedia, the free encyclopedia

[10] Chesbrough H 2006 Open business models: how to thrive in the innovation landscape. Harvard Business School Press, Boston

[11] Ernst \& Young L L P 2020 Healthcare goes mobile: Evolution of teleconsultation and e-pharmacy in new Normal, September 2020

[12] Shubham S, Basel K, Rob L and Zachary G 2020 The next wave of healthcare innovation: the evolution of ecosystems, McKinsey \& Company

[13] Ulrich P, Niklas K, Julian K, Evangelos A and Andreas K 2021 The untapped potential of ecosystems in health care, Boston Consulting Group

[14] Elliot S, 2013 Digital hospital 21st century, Second WHO Global Forum on Medical Devices, November 22, 2013

[15] Osterwalder A and Pigneur Y 2010 Business model generation: a handbook for visionaries, game changers, and challengers. Wiley, Hoboken

[16] KPMG, 2017 Supply chain Big data Series-Part, March 2017,kpmg.com.au

[17] Sebastian H, Tobias R, Eric KC, Markus B and Helmut K 2020 The digital transformation of the healthcare industry: exploring the rise of emerging platform ecosystems and their influence on the role of patients, Business Research

[18] Pfannstiel M A and Rasche C (eds) 2017 Service business model innovation in healthcare and hospital management models, strategies, toold. Springer, Berlin 
[19] Yu S, Fei H, Mingde Q, Wanxuan L and Ying J 2021 A dataenabled business model for a smart healthcare information service platform in the era of digital transformation. $J$. Healthc. Eng. 2021, Article ID 5519891

[20] Viswanadham N 2018 Performance analysis and design of competitive business models. Int. J. Prod. Res. 56(1-2): 983-999. https://doi.org/10.1080/00207543.2017.1406171

[21] Viswandham N and Kameshwaran S 2013. EcosystemAware Global Supply Chain Management. World Scientific Publishing Company

[22] Mark W. Johnson, Clayton M. Christensen,and Henning Kagermann, Reinventing Your Business Model, Harvard Business Review, December 2008

[23] Ramakrishna S V, Priya A, Vasantha K 2015 Doing well to do good: business model innovation for social healthcare, chapter 10 in business models and modelling; Volume 33; Advances in Strategic Management, editors C. Baden-Fuller and V. Mangematin; Emerald Press

[24] Viswanadham N and Samvedi A 2013, Supplier Selection Based on Supply Chain Ecosystem, Performance and Risk Criteria, IJPR, August 2013

[25] Viswanadham N 2015 Structural holes and supply chain orchestration, Heavy Haulers 1(2), 82-93

[26] Yesha P, Elonnai H, Amber S and Udbhav T, Artificial intelligence in the healthcare industry in India, The Centre for Internet and Society, India

[27] Weill P, Malone TW, Urso D, Victoria H G and Stepha W 2005 Do some business models perform better than others? A study of the 1000 largest US firms, Working Paper No. 226, 2005, http://ccs.mit.edu/papers/pdf/wp226.pdf 